\title{
CURTAILED NUMBER AND REDUCED CONTROLLER MOVEMENT OPTIMIZATION ALGORITHMS FOR REAL TIME VOLTAGE/REACTIVE POWER CONTROL
}

\author{
S.A.SOMAN K. PARTHASARATHY D. THIKARAM \\ Electrical Enginecrimg Department \\ Indian Institute of Sciclice \\ Bangalore, India.
}

\begin{abstract}
This paper addresses the problem of curtailing the number of control actions and minimizing controller movements for real-time voltage/reactive power control. Algorithms are proposed to identify the most effective subset of control actions and to minimize controller movements. An algorithmic objective function appropriate for the treatment of system security and economy is also proposed. It eliminates trial and error adjustments of weightage factors for a combined objective. A single parameter decides the priority between movement of controls and gains in security as well as loss reduction. The algorithms are compared and contrasted with SLP and SQP techniques by means of two practical systems.
\end{abstract}

Keywords: OPF, voltage control, Real-time control, reactive power control.

\section{INTRODUCTION}

The development of Reactive Power Optimization(RPO) and control algorithms for real time applications is of prime importance for optimal and secure operation of power systems. The Optimal Power Flow (OPF) is perhaps the most complex on-line EMS advanced application function under development. Significance of real time optimal operation of power systems stems from security and economic considerations.

For secure power system operation, redundancy is required in the system for driving it to the normal state in case of credible contingencies/emergencies. Redundancy in the power system w.r.t. reactive power control and optimization implies avajlability of alternate configurations of the network with acceptable reactive power flows, in terms of line MVA loading, bus voltages, MVA loading of transformers and generators. These factors determine the steady state security of the power system. With ever increasing load demands and system expansion costs, redundancy in the system has to be sacrificed. Instead, optimal use of reactive power control resources for maintaining real time steady state security becomes essential.

Econonic benefits can be achieved by rcal time optimal control of power systems. This demands optimal scheduling of generation based on availability and generation cost (Unit commitment and

94 WM 254-3 PWRS A paper recommended and approved by the IEEE Power System Engineering Committee of the IEEE Power Engineering Society for presentation at the IEEE/PES 1994 Winter Meeting, New York, New York, January 30 - February 3, 1994. Manuscript submitted August 27, 1992; made available for printing

December 6, 1993. active power optimization problems) and optimal allocation of the reactive power resources for security or economic objective.

The "state of the art" OPF/RPO algorithms assist the operator in deciding the best possible gains that can be achieved for security or economy oriented objectives. However, with increasing system sizes and controls, variety in the type of controls, their distributed nature and the real time restrictions on the number and the amount of control, the operator is faced with the formidable task of identifying the effective subset of control actions that can be implemented. In this sense, requirement of the real time OPF/RPO algorithm is to aid the operator in selecting the minimum number of effective controls. In addition, the operator has to maintain adequate reserves to deal with probable contingencies. Glavitsch et. al. in [2] and Tinney et. al. in [1] outline the desirable features of a real time OPF algorithm. Glavitsch and Bacher emphasise the requirements of high computational speed, robustness and reduced controller movements. We quote from [2], " The OPF assigns an optimal value to each possible control variable. Assuming that there is a large number of possible control variables, the OPF algorithm would move most of them from actual state to the optimal state. However, a practical real-time realization of this optimal state is not possible since the operator cannot have, e.g., hundred generator voltages be moved to different settings within a reasonable time. Only the most effective subset should be moved, which means that within the OPF the algorithmic problem of moving the minimum number of controllers with maximum effect has to be solved." Tinney et. al.[1] have addressed the need to curtail the number of control actions to meet the real time requirements and the difficulties faced in the selection of minimal number of effective controls in order to achieve this. We quote, "There is no way to select a subset of the most important control actions from total set of control actions. in an OPF solution. The actions are not ranked and importance of an action is not necessarily related to its magnitude. Even if a given number of best actions could be selected from a OPF output, the correct magnitude for the selected actions would be unknown because they would have to be readjusted to compensate for the omitted actions." The paper [1] proposes two possible formulations to meet these requirements. In the first formulation, the total number of control actions of each class were specified ( refer [3] for similar OPF algorithm development). However, they also point out that setting this limit in advance could cause the cost of the solution to be unacceptably ligh. The problem would also be well defined if a limit were specified on the increase in cost above minimum attainable, with all controls in use; but this could cause too many controls to be used (for interesting related reading refer to the discussion of [4]).

A significant feature that differentiates a real time OPF from standard optimization problem is that suboptimal solution is acceptable for the former because of the real world constraints. Glavitsch points out in [2] that consideration of data uncertainities can be used to speed up the algorithm, for e.g., if the accuracy of 
a large generator output power measurement is about five MW, making a computation with an accuracy of one MW is useless and consumes unnecessary computing time. As per Tinney's opinion [1], developing a method for curtailed control actions appears to be exceptional and coping with it will require unusual approaches.

We have not observed in the literature any appropriate theory for reducing the number of control actions and its correlation to the increase in cost. An algorithm to reduce the number of control actions based on sensitivity analysis and space \& time decomposition of controls has been proposed in [3]. However, the algorithm cannot identify the most effective subset of controls. The heuristic judgement of the designer plays an important role in this process. This paper for the first time presents the appropriate algorithms with strong theoritical support and negligible heuristics for achieving the important goals of minimizing the amount of controller movement $\left(\|\Delta u\|_{2}=\min \right)$ and curtailing the number of controllers in real time for $\mathrm{VQ}$ optimization. The proposed algorithm implicitly strikes a balance between

- reducing voltage infeasibilities.

- reducing MW losses in the system.

- reducing the number of controls.

The above three distinct and contrasting requirements are controlled via a single parameter $\epsilon$, resulting in simple and efficient algorithms. The approach is faster than standard optimization methods. The proposed algorithms are robust, have fast and easy convergence properties. The objective function has an automatic balancing feature to ensure security and reduction of active power losses in the system. The algorithmic nature of the objective function removes the requirement of trial and errors, with varying weightage factors for combined economy and security oriented objective. Comparitive evaluation with Sequential Linear Programming(SLP) and Sequential Quadratic Programming(SQP) algorithms is also presented, wherein complete optimization is performed, to study the effects of curtailing the number of controls and corresponding increase in cost function.

In the following sections, we first identify the important characteristics of the OPF algorithm that can be exploited for development of fast, robust and reliable algorithms. Then the essential optimization theory required for minimal control action algorithms is presented, as no such theory has been presented earlier. It is followed by the algorithms for minimizing and curtailing the control actions for security cum economy oriented objectives. Simulations for practical power systems are presented.

\section{REAL TIME VQ OPTIMIZATION}

The secure and optimal operation of power systems require the following:

- Optimal Power Flow.

- Corrective rescheduling.

- Corrective switching.

- VQ optimization.

The cycle time for a classical OPF is about $60-90 \mathrm{~min}$ [11]. With changes in either the load profile or the network topology, corrective rescheduling of $P$ and $Q$ variables as well as corrective switching actions need to be carried out.
VQ optimization and control is required to minimize losses, provide adequate reactive power margins, improve the voltage profile and to avoid voltage critical problems like voltage instability. The inputs to a VQ optimization problem are the system load and voltage profile, network information and the active and reactive controller settings obtained from the real time data base. The VQ optimization algorithm has to adjust the reactive power control variables to achieve one or more of the above objectives. To realize maximum economic benefits from $V Q$ optimization, a cycle time of $15 \mathrm{~min}$ is considered satisfactory [4] for an optimization and control frequency of $60 \mathrm{~min}$. However, for improvements in system security and stability, smaller cycle time for VQ optimization is required.

\section{VQ PROBLEM CHARACTERISTICS}

In this section typical VQ optimization problem characteristics are identified to enable the development of reliable, fast and efficient algorithms. Firstly, a VQ optimization problem is characterized by two distinct type of constraints viz., soft and hard constraints. We define them as follows.

Defn-1: A Hard constraint in an optimization problem is a constraint that has to be strictly satisfied. These constraints in a VQ optimization problem are device constraints, generator $Q$ limits and possible line loading limits.

Defn-2: A Soft constraint in an optimization problem is a constraint whose limits (lower and upper) can be characterized as "desirable" rather than "essential" and hence, need not be "necessarily" satisfied. These limits in a VQ optimization problem correspond to voltage bounds for the PQ-nodes. These constraints introduce an extra freedom which the optimization algorithm should be able to exploit. In general penalty function approaches and their variations, e.g., augmented lagrangian methods enforce these constraints in a soft way. However, the price is paid in terms of possible numerical instability and reduced computation speeds. The approach presented in this paper is not a penalty function approach. Specifically, in this paper we propose algorithms that are fast and numerically stable; they do exploit the softness of voltage constraints.

Secondly, an important feature of a VQ optimization problem is that the number of constraints (lower and upper voltage bounds, generator Q-limits, Q-injection variables and On Load Tap Changing transformer (OLTC) tap limits) far exceed the number of control variables. MVAR line loading constraints can also be considered in the above set. An optimization algorithm, while reducing voltage deviations should be able to exploit this redundancy in constraints for selecting the "best descent direction". The "best descent direction" is characterized mainly by the property of minimizing the voltage infeasibilities with minimum amount of control. The above ideas can be expressed within the mathematical framework of "best approximation" defined as follows.

Defn-3: A vector $x_{0}$ is a best approximation of the solution of the equations $f(x)=G$ ( $\mathrm{G}$ can be a vector or a matrix)[8], if for all $\mathrm{x}$, either

$$
\begin{array}{ll}
\text { 1. } & \|f(x)-G\|_{F}>\left\|f\left(x_{o}\right)-G\right\|_{F} \text { or } \\
\text { 2. } & \|f(x)-G\|_{F}=\left\|f\left(x_{o}\right)-G\right\|_{F} \text { and }\|x\|_{2} \geq\left\|x_{o}\right\|_{2}
\end{array}
$$

Part-1 of the definition is significant because it recognizes that infeasibilities cannot necessarily be reduced to zero; a significant aspect in enforcing soft constraints. This has been exploited to develop robust algorithms. Importance of part-2 arises from the fact that it ensures minimal norm solution in case of multiple min- 
ima having same cost. Specifically in VQ optimization, this can be used to guarantee minimum control action for minimizing the deviation of voltage profile of the system.

Finally, objective function evaluation, e.g., active power loss or power system voltage profile for a given control can be performed by a Power Flow(PF) program. As the network is very sparse, sparse computations drastically bringdown the required computational effort. Therefore, line-search based optimization algorithms are very suitable for OPF from computational viewpoint. Note this is in contrast with many other optimization problems, e.g., VLSI design, wherein computation of new objective function may require extensive simulations. In such cases, line-search methods become inefficient.

Thus, an efficient real time VQ optimization algorithm should incorporate the following characteristics.

- It should differentiate between hard and soft constraints, while being robust (numerically stable and fast).

- It should be able to exploit the redundancy and softness of the constraints to minimize control action.

- It should be predominantly a line-search based algorithm to facilitate simple and fast computing.

- Objective should reflect the requirements and the tradeoffs of real-time control

Various security or economy oriented objectives have been proposed in the literature for reactive power control. It has been pointed out in [3] that neither active power loss minimization nor uniform distribution of reactive reserves to generators operation in secondary control can meet the real-time objectives. If the OPF objective is active power loss minimization, the OPF results in high voltages in network. Also, reactive overloads appear at all generators electrically close to load centers. On the other hand, security oriented objective has a negative impact on system voltage profile. Therefore, a security cum economy oriented objective function is required in real-time with an implicit balancing feature to control the above mentioned effects. Also, the objective function should have the characteristic of implicitly reducing the required amount of control.

\section{FORMULATING A MINIMUM CONTROL ACTION PROBLEM}

The previous section outlined the utility of best approximate solution in defining a minimal control action problem. A more precise and desirable problem from minimum control point of view can be defined by considering a constrained non-linear minimization problem with objective function $f(u)$. Let $u^{o}$ represent the initial control vector and let $H=\{u: f(u)$ is a local minimum for the constrained problem\}. Then the minimal control action problem can be formulated as follows.

Find $u^{*} \epsilon H$ such that $\left\|u^{*}-u^{o}\right\|_{2} \leq\left\|u-u^{\circ}\right\|_{2}$ for all $u \in H$. Because of the difficulties involved in solving the above problem, we consider an iteration dependent non-linear security oriented objective function for RPO. At the $k_{-}$th iteration, the following optimization problem is attempted.

nuinimize for all $i \in \mathrm{PQ}$ nodes and $j \epsilon \mathrm{PV}$ nodes and lines with violated MVA flows having dominant MVAR loading

$\sum_{i}\left(v_{i}-v_{i}^{d e s^{k}}\right)^{2}+\sum_{j}\left(h_{j}-h_{j}^{d e s^{k}}\right)^{2}$

subject to $u^{\text {min }} \leq u \leq u^{\text {max }}$

$Q_{i}^{\min } \leq Q_{i} \leq Q_{i}^{\max }$ for all $i$ belonging to the set of PV-nodes. $-\sqrt{S_{l}^{\text {max }}-P_{\text {flow }}^{k^{2}}} \leq Q_{\text {flow }} \leq \sqrt{S_{l}^{\text {max }}-P_{\text {flow }}^{k^{2}}}$ for all $l$ be- longing to the set of violated line flows with dominant MVAR loading.

The element $h_{j}$ represents the $j_{-}$th element of the vector $h$ which contains all the generation Q-injection variables and the MVAR flow variables $\left(\mathcal{Q}_{\text {flow }}\right)$ for lines with violated MVA and dominant MVAR loading. $P_{\text {flow }}^{k}$ represents MW flow in the line $l$ at the $k_{-}$th iteration in either of the two directions. If real power limits are exceeded in a line, the reactive power control is unlikely to eliminate the violations of the line MVA loading limits. Such violations are corrected by resheduling the active power control variables [12]. Therefore, only those lines having MVA violations with significant MVAR loadings are considered in (1). The formulation is based on the assumption that changes in real power in the lines due to small changes in reactive power control variables are negligible. Let $b^{k^{T}}=\left[v^{\text {des } s^{k^{T}}}, h^{\text {des } s^{k^{T}}}\right]^{T}$. The elements of the vector $b^{k}$ depend on the system voltage profile, generator Q-injections, line MVAR flows andan iteration count $k$; they are set as follows. For all $i$ belonging to set of $\mathrm{PQ}$-nodes

if $v_{i}^{m i n} \leq v_{i}^{k} \leq v_{i}^{\max }$ then corresponding $b_{j}^{k}=v_{i}^{k}$

if $v_{i}^{k}>v_{i}^{\max }$ then corresponding $b_{j}^{k}=v_{i}^{\max }$

if $v_{i}^{k}<v_{i}^{m i n}$ then corresponding $b_{j}^{k}=v_{i}^{\text {min }}$

For all $i$ belonging to set of $\mathrm{PV}$-nodes

$$
\begin{aligned}
& \text { if } Q_{i}^{\text {min }} \leq Q_{i}^{k} \leq Q_{i}^{\text {max }} \text { then corresponding } b_{j}^{k}=Q_{i}^{k} \\
& \text { if } Q_{i}^{k}>Q_{i}^{\max } \text { then corresponding } b_{j}^{k}=Q_{i}^{\text {max }} \\
& \text { if } Q_{i}^{k}<Q_{i}^{\text {min }} \text { then corresponding } b_{j}^{k}=Q_{i}^{\text {min }}
\end{aligned}
$$

The elements of $b^{k}$ for MVAR line flow constraints considered in $h$ are set in a similar fashion. Note that as correction to the voltage profile is being done, reduced control action is implicit in the above objective. We also provide an economic bias to the security oriented objective by including active power loss reduction in the algorithm.

\section{THEORY FOR MINIMAL CONTROL ACTION}

The minimal control action problem for feasibility restoration has been precisely defined in the previous section. However, the load bus voltage vector $\left(v_{L}\right)$, generator Q-injection and MVAR line flow vector $(h)$ cannot be expressed explicitly as a function of $u$. Therefore, compact modelling of Dommel and Tinney [5] is used to linearize (1). Without loss of generality, we assume that the vector $b^{k}$ can be partitioned into two sub-vectors $b_{v}^{k}$ and $b_{h}^{k}$ corresponding to the constraint sets $v_{L}$ and $h$ respectively. Linearizing (1) at the $k_{-}$th iteration, we have

$\left[\begin{array}{l}\Delta v_{L}^{k} \\ \Delta h^{k}\end{array}\right]=\left[\begin{array}{c}S_{v}^{k} \\ S_{h}^{k}\end{array}\right] \Delta u=\left[\begin{array}{c}b_{v}^{k}-v_{L}^{k} \\ b_{h}^{k}-h^{k}\end{array}\right]$

Let $S^{k^{T}}=\left[S_{v}^{k^{T}}, S_{h}^{k^{T}}\right]$ and $b_{r}^{k^{T}}=\left[\left[b_{v}^{k}-v_{L}^{k}\right]^{T},\left[b_{h}^{k}-h^{k}\right]^{T}\right]$.

$b_{r}^{k}$ is a "restoration vector" with non-zero elements for violated load bus voltages, generator $\mathrm{Q}$-injections and MVAR line flows of vector $h$.

The above set of equations represent an overdetermined system. It is evident that $\Delta u$ must correspond to the "best approximation" for the linearized set of equations (2). Following theorem outlines the methodology of obtaining the best approximation. Theorem-1: The "best approximate" solution of the equations $A X=B$ is $X_{o}=A^{+} B . A^{+}$is the pseudo-inverse of $A[8]$ If $\mathrm{A}$ is $(\mathrm{m} \times \mathrm{n})$ matrix, $m>n$ and $\operatorname{null}(\mathrm{A})=0$, then $A^{T} A$ is nonsingular and $A^{+}=\left(A^{T} A\right)^{-1} A^{T}$. If $S^{k}$ is a full rank matrix, an efficient way to compute the best approximation is by QR factorization of $S^{k}$; a unique solution for $\Delta u$ exists that minimizes the infeasibilities at each iteration. However, in practice the overdetermined system need not be full rank; therefore the following two important questions arise. 
- Is the overdetermined matrix $S^{k}$ rank deficient?

- If yes, how do we then compute the best approximation for $\Delta u$ ?

The rank of a matrix $A$ and its pseudo-inverse can be computed by Singular Value Decomposition (SVD) of'A. An important mathematical result is given below:

Theorem-2: Let $A \in \Re^{m \times n}$, then there are orthogonal matrices $P$ and $Q$, such that $[7]^{\circ}$

$$
\mathrm{A}=Q\left(\begin{array}{ll}
\Sigma & 0 \\
0 & 0
\end{array}\right) P^{T}
$$

where $\Sigma=\operatorname{diag}\left(\sigma_{1}, \sigma_{2}, \ldots ., \sigma_{r}\right), \sigma_{1} \geq \sigma_{2} \geq \ldots . \geq \sigma_{r}>0$, $\sigma_{i}$ is the $i_{-}$th singular value and $r$ the rank of matrix A. It can also be proved that

1. $\|A\|_{F}=\sigma_{1}^{2}+\sigma_{2}^{2}+\ldots+\sigma_{n}^{2}$

2. for a $m \times n$ matrix $\mathrm{A}, m \geq n$ with singular values $\sigma_{1}, \sigma_{2}, \ldots, \sigma_{n}$; $\sigma_{1} \geq \sigma_{2} \geq \ldots \geq \sigma_{n}$, a $(m \times n)$ matrix $\mathrm{B}$ with rank $\mathrm{r}$ and $A^{\prime}$ defined

$$
\text { as }
$$$$
A^{\prime}=Q\left(\begin{array}{c}
\Sigma^{\prime} \\
0
\end{array}\right) P^{T}
$$

where $\Sigma^{\prime}=\operatorname{diag}\left(\sigma_{1}, \sigma_{2}, \ldots ., \sigma_{r}\right), \sigma_{1} \geq \sigma_{2} \geq \ldots . \geq \sigma_{r}>0$ $\left\|A-A^{\prime}\right\|_{F}=\min \|A-B\|_{F}$ and

3. the best approximation for an overdetermined system is

$$
X^{o}=A^{+} b=P\left(\begin{array}{c}
\Sigma^{-1} \\
0
\end{array}\right) Q^{T} b
$$

From the above properties, we can construct a matrix $A^{\prime}$ of rank $\mathrm{r}$, which is nearest to $\mathrm{A}$ in its frobenius norm. The above results are significant because of the fact that Gaussian Elimination cannot be used in practice for determination of the rank of a matrix, as numerical computations are approximate and as such produce non-zero diagonal entries, even in case of rank deficiency. As such in practice, possibility of rank deficiency (and also the probable rank) and the nearest rank deficient matrix are computed using SVD in the following manner [7].

Let the elements of $S^{k}$ be accurate to quantities of order $\epsilon$. Find r such that $\sigma_{r+1}^{2}+\sigma_{r+2}^{2}+\ldots+\sigma_{n}^{2}<\epsilon^{2}$. Then, the probable rank of $S^{k}$ is r. By property $2 \& 3$ of Theorem-2, with $\sigma_{r+1}$ to $\sigma_{n}$ set to zero, nearest rank deficient matrix $S^{k^{\prime}}$ and the corresponding best approximate solution $\Delta u$ can be computed. In actual implementation, the matrices $\mathrm{P}$ and $\mathrm{Q}$ need not be stored explicitly. A variation of the above method used to compute the best approximation for possible rank deficient matrix is to set all $\sigma_{i}<\epsilon^{\prime} \sigma_{1}$ to zero [6]. An important observation is that the best approximation $\Delta u^{k}$ so determined with non-zero $\epsilon$, minimizes infeasibilities with minimum control action i.e., if there exists a control $u^{\prime}$ such that $\left\|b_{r}^{k}-S^{k} \Delta u^{\prime}\right\|_{2}=\left\|b_{r}^{k}-S^{k} \Delta u^{k}\right\|_{2}$, then $\left\|\Delta u^{k}\right\|_{2}<\left\|\Delta u^{\prime}\right\|_{2}$. Infact, in practice, parameter $\epsilon$ can be used to restrict the amount of control action. It is proved in appendix- $D$ that the second norm of the control action $\left(\|\Delta u\|_{2}\right.$ ) obtained from best approximate solution of (2) decreases with the increase in control parameter $\epsilon^{\prime}$. Our experience suggests 0.001 to 0.005 as a suitable range for $\epsilon^{\prime}$. This results in matrix $S^{k \prime}$ close to the original matrix $S^{k}$; the change in frobenius norm is around $0.001 \%$.

\section{ALGORITHM FOR MINIMAL CONTROL ACTION}

As VQ control is a nonlinear optimization problem and the amount of control $\Delta u$ suggested after linearization may not be feasible, an iterative procedure incorporating a line-search along the direction of best approximation to minimize a selected objective needs to be formulated. The choice of objective function for line-search is not unique. Therefore, there exists an additional flexibility in the algorithm, as conflicting requirements like se- curity and economy, for e.g., minimizing the amount of control action versus minimizing the active power losses can be handled simultaneously by this procedure. An estimate of the change in active power loss along the best approximate direction is derived in appendix-B. As has already been pointed out line-search is computationally efficient for VQ optimization. It will involve repetitive PF computations wherein strict enforcement of hard Q-generation constraints is done. The developed procedure will have inherent numerical stability because of the use of orthogonal matrices and explicit treatment of rank deficient matrices. Also note that such an algorithm will enforce the voltage constraints in a soft manner without recourse to penalty function or augmented lagrangian methods. A model algorithm is now presented:

AG1: Feasibility restoration and loss reduction algorithm with minimum control action

STEP 0: Set iteration count $k=0$. Select $\epsilon^{\prime} \& \epsilon$.

STEP 1: Do a PF and determine $P_{L}^{o} \&$ restoration vector $b_{r}^{o}$.

STEP 2: If Voltage and generator Q-constraints satisfied or if $k>0$ and $P_{L}^{k-1}-P_{L}^{k}<\epsilon$, stop.

STEP 3: Compute $S^{k}$ (appendix-A).

STEP 4: Compute nearest rank deficient approximation $S^{k^{\prime}}$ to $S^{k}$.

STEP 5: Compute $\Delta u^{k}=S^{k^{+}} b_{r}^{k}$

STEP 6: Set $s^{k}=\Delta u^{k}$

STEP 7: Do a line search along $s^{k}$ to minimize $P_{L}$. Let the optimal step size be $\alpha^{k}$ where $0 \leq \alpha^{k} \leq 1$. Set $u^{k+1}=u^{k}+\alpha^{k} s^{k}$, $P_{L}^{k+1}=P_{L}$ and compute $b_{r}^{k+1}$.

STEP 8: Increment the iteration count $k=k+1$. Go to step 2 .

If the voltage profile is not satisfactory $t^{\prime}, 3 n \epsilon^{\prime}$ should be reduced in the next VQ cycle. To provide an economic bias during the line-search to the security oriented objective, we minimize active power losses. The algorithm automatically strikes a balance between security and economy in the corresponding order. When the system profile changes drastically, complete optimization (OPF) should be performed. The merits and desirable features of the algorithm (AG1) are:

1. Automatic balancing features for the conflicting requirements of attaining the maximum possible economic gains versus minimizing the control action and maintaining the security of the system.

2. Rapid convergence to the optimal solution (table-II). This results because of marginal changes in the singular values of the sensitivity matrix. This may also be used to speed-up the algorithm by avoiding SVD at each iteration.

3. Accurate modelling of the non-linearities of the system as repeated PF's are performed during the line-search.

4. Unimodal behaviour of the active power losses of the system. Thus, the possibility of the multiple minima along the search direction are eliminated.

\section{Curtailing the number of control actions}

Curtailing a variable movement, in it's natural sense, can be thought of as restraining the movement of the variable in a physical or a numerical process. In a numerical process, variables will tend to move marginally, from their initial values because of the "small" nonzero terms introduced in the numerical computation. However, such a movement will have negligible influence on the objective function. Therefore, we curtail the movement of a variable $\left(x_{i}\right)$, if

$$
\left|x_{i}^{*}-x_{i}^{o}\right|<\epsilon 1 \&\left|f\left(x_{i}^{*}\right)-f\left(x_{i}^{o}\right)\right|<\epsilon 2
$$

where superscripts $*$ and $o$ denote the final and initial values re- 
spectively. The choice of $\epsilon 1$ and $\epsilon 2$ will depend on the uncertainity in the real time data base (obtained by state estimation) and the accuracy of the transducers used. Suitable values of $\epsilon 1$ and $\epsilon 2$ are suggested in the result section. For a VQ optimization problem a subset of variables for strict curtailment $(\epsilon 1=0)$ can be identified as follows.

1. Compute the loss derivatives $g^{k^{T}}=\left[\frac{\partial P_{L}}{\partial u_{1}}, \frac{\partial P_{L}}{\partial u_{2}}, \ldots, \frac{\partial P_{L}}{\partial u_{n c}}\right]^{T}$.

2. Compute $s^{k}=S^{k^{+}} \times b_{r}^{k}$, the minimal norm vector to minimize infeasibilities.

3. Now if, $g_{i}^{k}>0$ and $s_{i}^{k}<0$ or $g_{i}^{k}<0$ and $s_{i}^{k}>0$, then the $i_{-}$th control action reduces both the MW losses and the voltage infeasibilities. If however, $g_{i}^{k}$ and $s_{i}^{k}$ are of same sign, it implies that the $i_{-}$th control reduces the voltage infeasibilities but increases the MW losses. In such a case, the corresponding control can be eliminated by setting $s_{i}^{k}=0$. Notice that the choice of controllers used to attain the objective is not unique. The outlined procedure automatically identifies the most effective subset of controls to reduce active power losses as well as voltage infeasibilites. The effect of such a measure is most evident in case of the Q-injection variables (SVC's and capacitor banks) where $s_{i}>0$ and $g_{i}>0$ will imply that losses increase with increase in capacitive injection. Similarly $s_{i}<0$ and $g_{i}<0$ will imply increase in losses and reduction of infeasibility with increase in inductive compensation. An estimate of the change in active power loss along the modified search direction is derived in appendix-B. An improved algorithm(AG2) biased for active power loss reduction with curtailed controller actions is presented below.

AG2: Curtailing the number of controls for voltage profile correction and active power loss reduction

STEP 0: Select $\epsilon, \epsilon 1, \epsilon 2, \epsilon 3, \epsilon^{\prime}$.

Set for all $j=1, n c$; alossvar $(j)=0$. The variable alossvar $(j)$ accumulates an estimate of change in $P_{L}$ due to $j_{-}$th controller movement using piecewise linear approximation for loss function.

STEP 1: Do a PF and determine $P_{L}^{o} \&$ restoration vector $b_{\tau}^{\circ}$. STEP 2: If Voltage and generator Q-constraints satisfied or if $k>0$ and $P_{L}^{k-1}-P_{L}^{k}<\epsilon$, go to step 10 .

STEP 3 to STEP 6: same as in AG1.

STEP 7: If $g_{i}^{k}>0$ and $s_{i}^{k}>0: s_{i}^{k}=0$.

If $g_{i}^{k}<0$ and $s_{i}^{k}<0: s_{i}^{k}=0$.

STEP 8: [Line search] $i=0$. Select $\alpha_{1}^{k}$.

REPEAT

$$
\mathrm{i}=\mathrm{i}+1
$$

$u_{i}^{k}=u^{k}+\alpha_{i}^{k} s^{k}$

For all $\mathrm{j}=1, \mathrm{nc}, \operatorname{alossvar}(\mathrm{j})=\operatorname{alossvar}(\mathrm{j})+g_{j}\left(u_{i-1}^{k}\right)\left(\alpha_{i}^{k}-\alpha_{i-1}^{k}\right) s_{j}^{k}$. Select $\alpha_{i+1}^{k}>\alpha_{i}^{k}$

UNTIL $\left(P_{L}\left(u_{i-1}^{k}\right)-P_{L}\left(u_{i}^{k}\right)>\epsilon 3\right.$ AND $\left.\alpha_{i+1}^{k} \leq 1\right)$

STEP 9: $k=k+1 ; u^{k}=u_{i}^{k-1}$. Compute $b_{r}^{k}$. Go to step 2.

STEP 10: Do for $j=1, n c ;$ if $\left|u_{j}^{k}-u_{j}^{o}\right|<\epsilon 1$ and $|\operatorname{alossvar}(\mathrm{j})|<\epsilon 2$; curtail the control.

STEP 11: Stop.

\section{SLP AND SQP ALGORITHMS}

To evaluate the effects of minimizing control action, curtailing the number of controls and the use of algorithmic objective function on the sub-optimality of the solution, SLP and SQP algorithms have been implemented. Constrained active power loss minimization is used as the reference. The other motivation for these implementations is to evaluate the effects of exploitation of softness of the voltage constraints on the total infeasibilities and the MW losses at the optimal point. Finally, these studies have been beneficial in evaluating the numerical performance of SLP and SQP algorithms. Reduced model formulation (refer appendix-A) of Dommel and Tinney [5] have been used in the sensitivity computations. The SLP and SQP algorithms are detailed in appendix-C.

\section{TEST RESULTS}

A realistic assessment of the algorithms will require repetitive VQ optinization using a day's load curve and the snapshots of the control variables on that day. In absence of the load curve information, a more conservative estimate can be obtained by comparing the performance of AG1, AG2, SLP and SQP algorithms. However, in practice as the load profile varies gradually and the results of the previous cycle of VQ optimization are available; convergence and speed characteristics of the algorithms in practice will be much better compared to the simulations presented. The results of simulations on practical 89 and 319 nodes systems are summarised below.

In the systems considered, less than $2 \%$ change in generator voltages, 0.75 MVAR change in capacitor banks and less than half the transformer tap movement is curtailed, if the effect on losses is less than 0.1 MW.

\section{9-bus system}

This system comprises of $400,220,132,66$, and $33 \mathrm{KV}$ voltage levels. There are 12 generators, 21 switchable VAR compensators and 19 tap changing transformers. Voltage feasibility range for the PQ-nodes is between $95-105 \%$. Comparitive evaluation of SLP, SQP, minimal control action and reduced controller algorithms are presented in table-I.

TABLE-I: Results for the $\mathbf{8 9}$ bus power system

\begin{tabular}{|l|c|c|c|c|c|}
\hline & $\begin{array}{c}\text { INITIAL } \\
\text { STATE }\end{array}$ & SLP Alg. & SQP Alg. & AG1 & AG2 \\
\hline$P_{L}$ & 144.67 & 113.36 & 111.74 & 129.21 & 119.25 \\
\hline$\Delta P_{L} \%$ & - & -21.64 & -22.76 & -10.68 & -17.57 \\
\hline Comp & 140 & 458.575 & 458.12 & 145.27 & 281.35 \\
\hline$N_{v}$ & 49 & 3 & 2 & 22 & 5 \\
\hline$S_{v}$ & 4.480 & 0.024 & 0.03 & 0.553 & 0.103 \\
\hline$\mu_{v}$ & 0.919 & 1.015 & 1.022 & 0.981 & 0.998 \\
\hline$\sigma_{v}$ & 0.081 & 0.029 & 0.028 & 0.044 & 0.037 \\
\hline
\end{tabular}

First, it is observed from table-1 that minimal control action algorithm (AG1) drastically brings down the control action with significant reduction in MW losses and improvement in voltage profile. For e.g., it is observed that total compensation increases from 140 MVAR to 145 MVAR with AG1. As SLP and SQP algorithms have MW loss minimization as their primary objective, reduction in MW losses is more significant for these algorithms, but at the cost of large control action. For e.g., Q-compensation in the system is increased from 140 MVAR to 458 MVAR. Secondly, it has been found by simulations that almost $34 \%$ of the controls could be curtailed using AG2. The performance of AG2 is better than that of AG1 in all respects. The primary reason for this is that AG1 reduces capacitive compensation (because of the negative elements introduced in the search direction for the capacitive variables). However AG2 inhibits the reduction of capacitive injections ( $g_{i}^{k}$ and $s_{i}^{k}$ have same sign) and therefore, in totality improves the results. Finally, it is observed that tuned SLP algorithm's performance is comparable to that of the SQP algorithm performance; SLP algorithm is faster than SQP algorithm. The algorithms have been tested on a VAX-8810 computer. Computational effort required for the SLP, SQP, AG1 and AG2 algorithms is approximately $21,41,15$ and 16 seconds(CPU time) respectively. The value of the control parameter $\epsilon^{\prime}$ used in the above simulations is 0.005 . The effect of control parameter value on the control action suggested for AG1 and the convergence characteristics are tabulated in table-II. 
TABLE-II: Effect of control parameter $\epsilon^{\prime}$ on amount of control action

\begin{tabular}{|l|cc|cc|cc|}
\hline iter & \multicolumn{2}{|c|}{$\epsilon^{\prime}=0.005$} & \multicolumn{2}{c|}{$\epsilon^{\prime}=0.0025$} & \multicolumn{2}{c|}{$\epsilon^{\prime}=0.001$} \\
\hline & LOSS & $\|\Delta u\|_{2}$ & LOSS & $\|\Delta u\|_{2}$ & LOSS & $\|\Delta u\|_{2}$ \\
\hline 0 & 144.67 & 0.9629 & 144.67 & 1.7884 & 144.67 & 2.9369 \\
\hline 1 & 135.05 & 0.5572 & 129.14 & 0.8324 & 129.22 & 1.3213 \\
\hline 2 & 129.82 & 0.4023 & 125.10 & 0.8211 & 124.19 & 1.0707 \\
\hline 3 & 129.21 & 0.4019 & & & & \\
\hline
\end{tabular}

From table-II, it can be observed that as the value of control parameter is decreased, the corresponding amount of maximum possible control action increases while the losses decrease. Note that this behaviour is consistent with the lemma proved in appendix-D. Thus, $\epsilon^{\prime}$ parameter controls the amount of control. The table also shows that the convergence is steady and takes place in very few iterations. Irrespective of the value of $\epsilon^{\prime}$ used, no oscillations are observed during convergence. The steady reduction in $\|\Delta u\|_{2}$ indicates the steady decrease in the amount of maximum control needed, and thus demonstrates the utility of the algorithm.

\section{9 bus system}

This system comprises of $400,220,132$ and $66 \mathrm{KV}$ lines. There are 22 generators, $42 \mathrm{Q}$-injection variables and 24 tap changing transformers in the system. Voltage feasibility range for all the buses is from $95 \%$ to $105 \%$ of the nominal voltage. Results for the system are presented in table-III. No oscillations were observed during convergence to the final state. As can be observed, most of the earlier observations hold for this system also. As much as $50 \%$ controls have been curtailed by algorithm AG2 with only $1.8 \mathrm{MW}$ increase in power losses above the SQP results. Similarly, performance of AG1 is also satisfactory. Computational effort required for the SLP, SQP, AG1 and AG2 algorithms is approximately 70 , 156,50 and 75 seconds(CPU time) respectively.

TABLE-III: Results for 319 bus system

\begin{tabular}{|l|c|c|c|c|c|}
\hline & $\begin{array}{c}\text { INITIAL } \\
\text { STATE }\end{array}$ & SLP Alg. & SQP Alg. & AG1 & AG2 \\
\hline$P_{L}$ & 252.91 & 227.02 & 231.08 & 237.23 & 232.84 \\
\hline$\Delta P_{L} \%$ & - & -10.35 & -8.2 & -6.2 & -7.93 \\
\hline Conpen & 5 & 245.44 & 271.54 & 167.85 & 199.86 \\
\hline$N_{v}$ & 136 & 33 & 14 & 40 & 25 \\
\hline$S_{v}$ & 7.605 & 0.719 & 0.212 & 0.934 & 0.525 \\
\hline$\mu_{v}$ & 0.950 & .994 & 1.000 & 0.985 & 0.993 \\
\hline$\sigma_{v}$ & 0.058 & 0.032 & 0.029 & 0.033 & 0.031 \\
\hline
\end{tabular}

\section{CONCLUSIONS}

Algorithms were proposed to minimize the number of control actions as well as controller movements, in order to realize realtime objectives in voltage/reactive power control. These algorithms represent systematic procedures to improve the state of the power system considering at the same time practical constraints such as feasible and most effective controller movements. The cost of reduction/curtailment of controller movements is the suboptimality of the solution. With growing system sizes, it is impossible for the operator to identify the most effective subset of controls which result in maximum gains while meeting real world constraints. We feel these algorithms represent a significant advancement towards reducing operator dependency in $\mathrm{OPF}$, which is essential for real-time applications. The algorithms are based on sound mathematical and numerical techniques; they are robust, fast and easy to implement. A single parameter decides trade offs between movement of controls, number of controls and gains in security as well as loss reduction.

\section{LIST OF SYMBOLS}

$\mathrm{n}$ - size of the power system network.

$S_{l}^{\text {max }}$ - maximum permissible MVA for the $l_{-}$th line.

$P_{\text {flow }}^{k}-$ active power flow in the $l_{-}$th line in either of two directions at $k_{-}$th iteration.

$Q_{\text {flow }}^{k}$ - reactive power flow in the $l_{-}$th line in either of two directions at $k_{-}$th iteration.

$v_{i}$ - voltage at bus $i$.

$h$ - violating MVAR line flows and generator Q-constraint equations vector.

$v_{L}^{k}$ - vector of load bus voltages at $k_{-}$th iteration.

$v^{i e e^{k}}$ - desired voltage vector for vector $v_{L}$ at $k_{-}$th iteration.

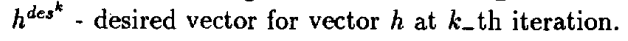

$Q_{i}$ - net reactive power injection at bus $i$.

$P_{L}$ - active power loss of the system.

$P_{s l}$ - slack bus active power injection.

Comp - total Q-compensation.

$g$ - load flow equations vector.

$g^{k}$ - power loss derivative at $k$-th iteration.

$N_{v}$ - number of violated PQ-node voltages.

$\mu_{v}$ - mean voltage of the system nodes.

$\sigma_{v}$ - standard deviation of voltages for the system.

$S_{v}$ - sum of absolute voltage infeasibilities.

$S_{v}^{k}$ - voltage sensitivity matrix at $k_{-}$th iteration.

$S_{h}^{k}$ - sensitivity matrix for constraints $h$ at $k_{-}$th iteration.

$S_{x}$ - senstivity matrix for state vector $x$.

$u$ - reactive power control vector.

$\|\cdot\|_{2}$ - Euclidean norm of a vector.

$\|\cdot\|_{F}$ - Frobenius norm of a vector.

$A^{+}$- pseudo-inverse of matrix A.

$\sigma_{i}-i_{-}$th singular value of a matrix.

ustep - restriction vector for the controller movements $(u)$.

nc - dimension of vector $u$.

w.r.t - with respect to.

r.h.s - right hand side.

Superscript $o$ denotes the initial point $\& k$ the iteration count.

\section{REFERENCES}

1. W. F. Tinney, J. M. Bright, K. D. Demaree, B.A. Hughes, 'Some deficiences in optimal power flow', IEEE-PICA Conf. Proc, Montreal Canada, May 1987, pp.164-169.

2. Hans Glavitsch, Rainer Bacher, 'Optimal power flow algorithms', Control and dynamic systems - Advances in theory and applications Vol 41: Analysis and control system techniques for electric power systems part 1 of 4 , edited by C.T. Leondes, Academic Press, 1991, pp. 135-206.

3. Milan Bjelogrlic, Milan S. Calovic, Borivoje S. Babic, P. Ristanovic, 'Application of newtons's optimal power flow in voltage/reactive power control', IEEE Transactions on Power Systems, Vol. 5, No. 4, November 1990 pp. 1447-1454.

4. M. A. El-Kady, B. D. Bell, V. F. Carvalho, R. C. Burchett, H. H. Happ, D. R. Vierath, 'Assessment of Real-time optimal voltage control', IEEE Transactions on Power Systems, Vol.PWRS-1, No. 2, pp.98-107, May 1986.

5. Hermann W. Dommel, William F. Tinney, 'Optimal power flow solutions', IEEE Transactions on Power Apparatus and Systems, Vol. PAS-87, No. 10, October 1968, pp. 1866-1875.

6. NAG Fortran Library Manual Mark-14, The Numerical Algorithms Group Ltd., Wilkinson House Jordan Hillroad, Oxford, UK, 1990 . 
7. G. W. Stewart, Introduction to matrix computations, Academic Press, 1973

8. Thomas. L. Boullion, Patrick. L. Odell,' Generalized inverse matrices', Wiley -Interscience, 1971

9. Philip E. Gill, Walter Murray, Margaret H. Wright, 'Practical Optimization', Academic Press, 1981.

10. L. Franchi, M. Innorta, P. Marannino, C. Sabelli, 'Evaluation of economy and/or security oriented objective functions for reactive power scheduling in large scale systems' IEEE Transactions on Power Apparatus and Systems, Vol. PAS-102, No. 10, October 1983 , pp. 3481-3487.

11. F. F. Wu, 'Real-time network security monitoring, assessment and optimization' Electrical Power \& Energy Systems, Vol. 10, No. 2, April 1988, pp. 83-100.

12. Brian Stott, Eric Hobson, 'Power system security control calculations using linear programming, Part I' IEEE Transactions on Power Apparatus and Systems, Vol. PAS-97, No. 5, Sep/oct 1978, pp. 1713-1720.

\section{APPENDIX-A}

\section{REDUCED MODEL FORMULATION}

As the active power injection variables except the slack bus power injection are fixed in RPO, $\Delta P_{L}=\Delta P_{s 1}$. On linearization of the power flow equations around the power flow solution, we obtain

$\left[\frac{\partial g}{\partial x}\right] \Delta x+\left[\frac{\partial g}{\partial u}\right] \Delta u=0$

$\Delta x=-\left[\frac{\partial g}{\partial x}\right]^{-1}\left[\frac{\partial g}{\partial u}\right] \Delta u$

$\Delta x=\left[S_{x}\right] \Delta u$

$\Delta P_{s l}=\left[\frac{\partial P_{a l}}{\partial x}\right]^{T} \Delta x+\left[\frac{\partial P_{s l}}{\partial u}\right]^{T} \Delta u$

$\Delta P_{s l}=\left[\left[\frac{\partial P_{s l}}{\partial x}\right]^{T}\left[S_{x}\right]+\left[\frac{\partial P_{s l}}{\partial u}\right]^{T}\right] \Delta u$

Similarly, $\Delta h=\frac{\partial h}{\partial x} \Delta x+\frac{\partial h}{\partial u} \Delta u$ $\Delta h=\left[\frac{\partial h}{\partial x} S_{x}+\frac{\partial h}{\partial u}\right] \Delta u$

Reduced gradients can be computed from the above expressions.

\section{APPENDIX-B}

\section{LOSS ESTIMATION FOR AG1}

Using second order approximation for $P_{L}(u)$, we have at the $k_{-}$th iteration

$\Delta P_{L}^{k}=\left[\frac{\partial P_{l}^{k}}{\partial u}\right]^{T} \quad \Delta u^{k}+\frac{1}{2} \Delta u^{k^{T}}\left[\frac{\partial^{2} P_{k}^{k}}{\partial u^{2}}\right] \Delta u^{k}$

Since $\Delta u^{k}=S^{k^{+}} b_{r}^{k}$, we have

$\Delta P_{L}^{k}=\left[\frac{\partial P_{I}^{k}}{\partial u}\right]^{T} S^{k^{+}} b_{r}^{k}+\frac{1}{2} b_{r}^{k^{T}}\left[S^{k^{+}}\right]^{T}\left[\frac{\partial^{2} P_{l}^{k}}{\partial u^{2}}\right]\left[S^{k^{+}}\right] b_{r}^{k}$

The above equation gives an estimate of the change in losses along $\Delta u^{k}$ given $b_{\tau}^{k}$. However, evaluation of the estimate as above is inaccurate as well as expensive. Therefore, line search is used in the proposed algorithm.

\section{LOSS ESTIMATION FOR AG2}

At the $k_{-}$th iteration, the search direction is given by $\Delta s^{k}=$ $D^{k} S^{k^{+}} b_{\tau}^{k}$, where $D^{k}$ is a diagonal matrix $\left(d_{i}^{k}\right)$ with $d_{i}^{k}=1$ if $g_{i}^{k}$ and $s_{i}^{k}$ have opposite signs and $d_{i}^{k}=0$ if $g_{i}^{k}$ and $s_{i}^{k}$ have same sign. With quadratic approximation of the loss function and the search direction computed as above, we have the estimate $\Delta P_{L}^{k}=\left[\frac{\partial P_{L}^{k}}{\partial u}\right]^{T} D^{k} S^{k^{+}} b_{r}^{k}+\frac{1}{2} b_{r}^{k^{T}}\left[S^{k^{+}}\right]^{T} D^{k^{T}}\left[\frac{\partial^{2} P_{L}^{k}}{\partial u^{2}}\right] D^{k}\left[S^{k^{+}}\right] b_{r}^{k}$

The expression is similar to the one given for $A G 1$, and the qualitative remarks for $\mathrm{AG} 1$ hold for $\mathrm{AG} 2$ also.

\section{APPENDIX-C}

\section{SLP ALGORITHM}

At the $k_{-}$th iteration of the SLP algorithm the following linear programming problem is solved.

$$
\begin{aligned}
& \min \Delta P_{s l}^{k}=\left[\frac{\partial P_{s l}}{\partial u}\right]^{T} \Delta u \text { subject to } \\
& \max \left(-u s t e p, u^{m i n}-u^{k}\right) \leq \Delta u^{k} \leq \min \left(u s t e p, u^{\max }-u^{k}\right) \\
& x^{\min }-x^{k} \leq \Delta x^{k} \leq x^{\max }-x^{k} \\
& h^{\min }-h^{k} \leq \Delta h^{k} \leq h^{\max }-h^{k}
\end{aligned}
$$

Expressions for $\Delta x$ and $\Delta u$ have been computed in (A1) and (A2).

\section{SQP ALGORITHM}

At the $k_{-} t h$ iteration of the SQP algorithm the following quadratic programming problem is solved.

$$
\begin{aligned}
& \min \left[\frac{\partial P_{\Delta l}}{\partial u}\right]^{T} \Delta u+\frac{1}{2}[\Delta u]^{T}\left[H^{k}\right][\Delta u] \\
& u^{\min }-u^{k} \leq \Delta u^{k} \leq u^{\max }-u^{k} \\
& x^{\min }-x^{k} \leq \Delta x^{k} \leq x^{\max }-x^{k} \\
& h^{\text {min }}-h^{k} \leq \Delta h^{k} \leq h^{\max }-h^{k}
\end{aligned}
$$

The matrix $H^{k}$ is a positive definite quasi newton Hessian approximation to the Hessian of the augmented lagrangian function at the $k_{-}$th iteration. The implementation utilizes E04VCF routine from the NAG library, which differs from the standard SQP algorithm of [9] in the following way. Firstly, at each iteration, strict feasibility is maintained for power flow constraints. Secondly, only inequality constraints exist in the reduced formulation solved by SQP method. Finally, the convergence of the algorithm is superlinear. For further discussions refer to $[9,10]$.

\section{APPENDIX-D}

Lemma: The second norm of the control action ( $\|\Delta u\|_{2}$ ) obtained from best, approximate solution of (2) decreases with the increase in control parameter $c^{\prime}$.

Proof: Let $S^{k}=Q\left(\begin{array}{cc}\Sigma & 0 \\ 0 & 0\end{array}\right) P^{T}$

Then, the corresponding best approximate solution for the control action $\left(\Delta u_{0}\right)$ is given by $\Delta u_{0}=S^{k^{+}} b_{r}^{k}$. By property-3 of Theorem 2 and the invariance property of second norm of a vector under linear transformation by an orthogonal matrix, we have

$$
\left\|\Delta u_{0}\right\|_{2}^{2}=\sum_{i=1}^{n}\left(\frac{q_{i}^{T} b_{x}^{k}}{\sigma_{1}}\right)^{2}
$$

where $Q=\left[q_{1}, q_{2}, \ldots, q_{m}\right]$.

Similarly, the second norm of the control action $\left(\Delta u_{\varepsilon}\right)$ obtained by using rank deficient approximation of the sensitivity matrix $\left(\epsilon^{\prime}>0\right.$ such that $\left.r<n\right)$ is given by

$$
\left\|\Delta u_{\epsilon} \prime\right\|_{2}^{2}=\sum_{i=1}^{r}\left(\frac{q_{i}^{T} b_{r}^{k}}{\sigma_{i}}\right)^{2}
$$

From (3) and (4), $\left\|\Delta u_{\epsilon}\right\|_{2}<\left\|\Delta u_{0}\right\|_{2}$. Similarly, for any two positive values of $\epsilon^{\prime}, \alpha$ and $\beta(\alpha<\beta)$, we have

$$
\left\|\Delta u_{\beta}\right\|_{2} \leq\left\|\Delta u_{\alpha}\right\|_{2}
$$

S. A. Soman received B.E degree in Electrical Engineering from the Regional Engineering College, Bhopal in 1989, M.E in Electrical Engineering from Indian Institute of Science, Bangalore in 1992. Currently he is working towards the Ph.D in Electircal Engineering department, IISc, Bangalore.

K. Parthasarathy(SM '89) received the B.Sc degree from Mysore University in 1956, the D.I.I.Sc, M.E and Ph.D in Electrical Engineering from Indian Institute of Science, Bangalore in 1959, 1961 and 1967 respectively. He is currently professor at Indian Institute of Science, Bangalore. His research interest s include computer aided protection, parallel processing, reactive power optimization and real-time control of power systems.

D. Thukaram(M '90) received the B.E degree in Electrical Engineering from Osmania University, Hyderabad in 1974, M.Tech in Integrated Power Systems from Nagpur Lniversity in 1976 and Ph.D from Indian Institute of Science,Bangalore in 1986. Since 1976, he has been with IISc, where he is currently Assistant Professor in the department of Electrical Engineering. His research interests include computer aided power system analysis,reactive power optimization, parallel processing and expert system applications to power systems. 